\title{
Proteomic identification of differentially expressed proteins associated with the multiple drug resistance in methotrexate-resistant human breast cancer cells
}

\author{
SIYING CHEN $^{1}$, JIANGXIA CAI ${ }^{1}$, WEIPENG ZHANG ${ }^{1}$, XIAOWEI ZHENG ${ }^{1}$, \\ SASA HU ${ }^{1}$, JUN LU ${ }^{1}$, JIANFENG XING ${ }^{2}$ and YALIN DONG ${ }^{1}$ \\ ${ }^{1}$ Department of Pharmacy, The First Affiliated Hospital of Medical College, ${ }^{2}$ Department of Pharmacy, \\ College of Medicine, Xi'an Jiaotong University, Xi'an, Shaanxi 710061, P.R. China
}

Received January 25, 2014; Accepted March 28, 2014

DOI: 10.3892/ijo.2014.2389

\begin{abstract}
Methotrexate (MTX), as a chemotherapeutic drug, is widely used in the therapy of several cancer types. The efficiency of drug treatment is compromised by the appearance of multidrug resistance (MDR), and the underlying molecular mechanisms remain incompletely understood. We investigated the mechanism of MDR in the MTX-induced breast cancer MCF-7 cells (MCF-7/MTX) using proteomic analysis. MCF-7 drug-sensitive cells (MCF-7/S) were exposed in progressively increasing concentrations of MTX to establish the drug-resistant cell line MCF-7/MTX. The biological characteristics of the cells were analyzed by MTT, flow cytometry, quantitative PCR, western blotting and the global protein profiles of MCF-7/MTX and MCF-7/S were compared using a proteomic approach. The resistance factor of MCF-7/ MTX cells was 64, and it possessed significant MDR. Seventeen differentially expressed proteins between MCF-7/ MTX and MCF-7/S cells were identified, seven proteins were upregulated and 10 proteins were downregulated in MCF-7/ MTX cells. We verified that the protein levels of nucleophosmin (NPM), $\alpha$-enolase (ENO1) and vimentin (VIM) were upregulated, and heterogeneous nuclear ribonucleoprotein (hnRNP C1/C2), phosphoglycerate mutase 1 (PGAM1) and proteasome subunit $\alpha$ type-2 (PSMA2) were downregulated in MCF-7/MTX cells. The mRNA levels of NPM, VIM, hnRNP C1/C2, PGAM1 and PSMA2 were consistent with the protein expressions, but the gene expression of ENO1 was slightly downregulated. Surprisingly, knockdown of $N P M$ by siRNA sensitized MCF-7/MTX cells to MTX and attenuated the multidrug resistance. The proteins identified, particularly
\end{abstract}

Correspondence to: Professor Yalin Dong, Department of Pharmacy, The First Affiliated Hospital of Medical College, Xi'an Jiaotong University, Xi'an, Shaanxi 710061, P.R. China

E-mail: dongyalin@mail.xjtu.edu.cn

Key words: breast cancer, multidrug resistance, methotrexate, proteomics, molecular targets
NPM provides new insights into the mechanism of MDR and is expected to become a crucial molecular target for breast cancer treatment.

\section{Introduction}

Breast carcinoma is a common malignant tumor among women, the incidence and mortality rates continue to rise rapidly worldwide along with lifestyle changes in diet and environmental factors (1). In Asian countries, the incidence of breast cancer increase doubled or even tripled in the past few decades, similarly, in China $\sim 20-30 \%$ of the new cases of breast cancer occurred in the past decade (2). Currently, most breast cancer patients receive chemotherapy, but the long-term stimulation of cytotoxic agents accelerate the occurrence of severe acquired resistance, especially multidrug resistance (MDR) has become an important cause of tumor recurrence after surgery and chemotherapy has failed (3). However, the molecular mechanisms of MDR are rather complicated, it involves in increased drug efflux, drug metabolic biotransformation and alteration of repair ability for DNA damage (4). Therefore, investigating the other potential mechanisms and screening the molecular targets of drug resistance have become key factors in the study of MDR of breast cancer.

Methotrexate (MTX), an antifolate antineoplastic, mainly prevents tumor cells growth and proliferation through potent inhibition of dihydrofolate reductase (DHFR) for purine and pyrimidine nucleotide biosynthesis and blockage of DNA replication. As a classical anticancer drug, it possesses strong efficacy and low price, which is widely used for the treatment of human leukemia, lymphoma, breast cancer, head and neck cancers and other solid tumors, both alone and in combination with other chemotherapeutics (5). Although antifolates are important and effective components of different chemotherapeutic regimens currently used for various cancer therapies, drug resistance frequently arises from the long-term use for treatment in clinic, and the molecular mechanism of antifolate-resistance in cancer cells is believed to be a multifactorial process, it relates to the loss of folate carrier (RFC) function, overexpression and mutation of DHFR and thymidylate synthase (TS), increased ATP-driven MDR efflux 
transporters, decreased polyglutamate synthase, the high expression of $\gamma$-glutamyl hydrolase and tetrahydrofolic acid (THF) (6). However, the MTX resistance is still an extremely complex process, and the mechanism of action may involve changes in a variety of pathways and expressions of different resistant proteins.

With the aim of gaining further insight into the molecular mechanisms of MDR and to discover new biological targets of MTX, the functional proteomic analysis using twodimensional gel electrophoresis (2-DE) and matrix-assisted laser desorption ionization time of flight mass spectrometry (MALDI-TOF-MS) were performed to identify the differential protein profiles between the MTX-resistant breast cancer cell line MCF-7/MTX and drug sensitive cell line MCF-7/S.

\section{Materials and methods}

Establishment of MCF-7/MTX cell line. The parental human breast cancer cell line MCF-7 was obtained from the Chinese Academy of Science (Shanghai, China). These cells were cultured in RPMI-1640 medium containing $10 \%$ fetal bovine serum (Gibco), $1 \%$ penicillin and streptomycin, at $37^{\circ} \mathrm{C}$ in a humidified atmosphere of $5 \% \mathrm{CO}_{2}$. The MTX-resistant cell line MCF-7/MTX was established from its sensitive cell line MCF-7/S by progressively increasing methotrexate (Shanxi Pude Pharmaceutical, China) over a period of 12 months. The culture condition was the same as the MCF-7/S cell line. The drug concentration was increased by 1.5 -fold at each step of resistance, from 4 to $220 \mathrm{nM}$. The cells were cultured in MTX of each step for at least 2 weeks before each experiment.

Drug sensitivity assay. The MTT assay (Sigma) was used to measure the drug sensitivity. Cells were seeded at a density of $4 \times 10^{4}$ cells per well in 96-well plate with each well containing $100 \mu \mathrm{l}$ medium. The cells were grown for $24 \mathrm{~h}$, then the culture medium was replaced with $200 \mu \mathrm{l}$ fresh medium containing MTX at different concentrations for $72 \mathrm{~h}$. The medium was removed and added with $180 \mu \mathrm{l}$ of RPMI-1640 and $20 \mu \mathrm{l}$ MTT (5 mg MTT/ml) and incubated for $4 \mathrm{~h}$. Following medium removal, $150 \mu \mathrm{l}$ of DMSO was added to solubilize the formazan. The absorbance was read at $490 \mathrm{~nm}$ on a microplate reader (BioTek ELx808, USA). The 50\% growth inhibitory concentration $\left(\mathrm{IC}_{50}\right)$ of drug was determined to evaluate the drug sensitivity. Resistance factor (RF) was calculated by the ratio of the $\mathrm{IC}_{50}$ values of MCF-7/MTX to MCF-7/S cells.

MDR detection. The sensitivity of MCF-7/S and MCF-7/MTX cells to doxorubicin (Zhejiang Haizheng Pharmaceutical, China), paclitaxel (Nanjing Sike Pharmaceutical, China), 5-fluorouracil (Shanghai Xudonghaipu Pharmaceutical, China), mitoxantrone (Shandong Luoxin Pharmaceutical, China), vinorelbine (Hangzhou Minsheng Pharmaceutical, China), gemcitabine (Jiangsu Haosen Pharmaceutical, China), cisplatin, docetaxel and pemetrexed (Shandong Qilu Pharmaceutical, China), were determined by MTT assay as described above, the $\mathrm{IC}_{50}$ value and $\mathrm{RF}$ for each drug were calculated.

Analysis of cell cycle distribution. MCF-7/S and MCF-7/MTX cells were seeded at a density of $4 \times 10^{5}$ cells per well into 6 -well plates and incubated for $24 \mathrm{~h}$. Cells were trypsinized, washed twice with cold PBS and fixed by $70 \%$ ethanol overnight at $4^{\circ} \mathrm{C}$, then washed and re-suspended with PBS containing RNase and propidium iodide. After $30 \mathrm{~min}$ in the dark, the cell cycle was analyzed using a flow cytometer (FACSCanto ${ }^{\mathrm{TM}} \mathrm{II}$, BD Biosciences, USA).

Preparation of proteins. MCF-7/S and MCF-7/MTX cells were harvested, washed with PBS and then lysed in the lysis buffer (8 M urea, $2 \mathrm{M}$ thiourea, 4\% CHAPS, $40 \mathrm{nM}$ Tris, $50 \mathrm{mM}$ DTT, $1 \mathrm{mM}$ PMSF, $0.5 \%$ (w/v) Bio-lyte and $0.5 \%$ IPG buffer) at $4{ }^{\circ} \mathrm{C}$ for $30 \mathrm{~min}$. After centrifugation at $14,000 \mathrm{rpm}$ for $40 \mathrm{~min}$ at $4^{\circ} \mathrm{C}$, the supernatants were purified by ReadyPrep2-D Cleanup kit (Bio-Rad, Hercules, CA, USA) according to the manufacturer's instructions and stored at $-80^{\circ} \mathrm{C}$ for use. The protein concentration was measured with Bradford method.

2-DE and image analysis. Each protein sample (800 $\mu \mathrm{g})$ was applied to $\mathrm{pH}$ 3.0-10.0 (non-linear $17 \mathrm{~cm}$ ) IPG strip (Bio-Rad) using a passive rehydration method. After rehydration for $16 \mathrm{~h}$, IEF was performed successively for $1 \mathrm{~h}$ at $250 \mathrm{~V}, 1 \mathrm{~h}$ at $1,000 \mathrm{~V}$, and $5 \mathrm{~h}$ at $10,000 \mathrm{~V}$ to give a total of $60 \mathrm{kvh}$ on an IPGphor. Then, the gel strip was equilibrated for 15 min with DTT and iodoacetamide, respectively. The second dimension was performed using 12\% SDS-PAGE until bromophenol blue reached the bottom of the gel. The gel was stained with sensitive colloidal Coomassie blue G-250 and scanned with UMax Powerlook 2110XL (Umax). Three independent experiments were made for each cell line to ensure the accuracy of the analyses. The images were analyzed by Imagemaster 2D Platinum (GE Amersham). The gel spot pattern of each gel was normalized and matched. The differentially expressed proteins between MCF-7/S and MCF-7/MTX in a given spot were calculated as the ratio of all the normalized spot values. Only spots that showed significant differences $( \pm>1.5$-fold, $\mathrm{P}<0.05)$ were selected for further analysis.

In-gel digestion. Differential protein spots were excised from the stained gels, destained in $30 \mathrm{mM}$ potassium ferricyanide/100 $\mathrm{mM}$ sodium thiosulfate $(1: 1 \mathrm{v} / \mathrm{v})$ for $20 \mathrm{~min}$ and washed with water. Then the spots were incubated in $0.2 \mathrm{M}$ $\mathrm{NH}_{4} \mathrm{HCO}_{3}$ for $20 \mathrm{~min}$ and freeze-dried. Each spot was digested with $12.5 \mathrm{ng} / \mathrm{ml}$ trypsin in $25 \mathrm{mM} \mathrm{NH}_{4} \mathrm{HCO}_{3}$ at $37^{\circ} \mathrm{C}$ overnight. The peptides were extracted three times with $60 \% \mathrm{ACN} / 0.1 \%$ TFA, and the extracts were pooled and dried in a vacuum centrifuge to complete desiccation at room temperature.

MALDI-TOF-MS analysis. The mass spectra were analyzed using MALDI-TOF-TOF instrument (4800 proteomics analyzer; Applied Biosystems). For protein identification, combined peptide mass fingerprinting (PMF) and the MS/ MS queries were performed automatically by searching the EBI database using the MASCOT search engine 2.2 (Matrix Science, UK). The database search was carried out with the following parameters: enzyme, trypsin; the allowance of one missed cleavage; carbamidomethylation was set as fixed modification; oxidation of methionine was allowed as variable modification; the peptide and fragment mass tolerance were set at $100 \mathrm{ppm} 0.4 \mathrm{Da}$, respectively. A GPS Explorer protein confidence index $\geq 95 \%$ was used for further manual 
Table I. Primer sequences for quantitative PCR.

\begin{tabular}{lllr}
\hline Gene & \multicolumn{1}{c}{ Forward primer (5'-3') } & \multicolumn{1}{c}{ Reverse primer (5'-3') } & Product size (bp) \\
\hline$M D R 1$ & GAGCCCATCCTGTTTGACTG & GCTGCCCTCACAATCTCTTC & 92 \\
$M R P 1$ & AAGGTGGACGAGAACCAGAA & AACAGGGCAGCAAACAGAAC & 110 \\
$B C R P$ & AGCAGGGACGAACAATCATC & GCCAATAAGGTGAGGCTATCA & 82 \\
$R F C$ & TCCTGTCCATCATCTACTTCTTG & AGTGCCTGTGCTGCCTTCT & 130 \\
$D H F R$ & TCTCCAAGACCCCAACTGAG & ATGTGAAAAGCCCGACAAT & 109 \\
$N P M$ & TGGCAGTGGAGGAAGTCTCT & ATCAAACACGGTAGGGAAAGT & 141 \\
$E N O 1$ & TCCCTTTGACCAGGATGACT & GACTTTGAGCAGGAGGCAGTT & 151 \\
$V I M$ & GAAGAGAACTTTGCCGTTGAAG & GAAGGTGACGAGCCATTTC & 100 \\
$h n R N P C 1 / C 2$ & GCTTTGCCTTCGTTCAGTATG & CCCGTTGAAAGTCATAGTCCA & 240 \\
$P G A M 1$ & GCTCCCCTTATCCAACAGAGTT & TTGCTTCTCCTCACTGGTCAT & 114 \\
$P S M A 2$ & CCAAGCAGAATGATGAAATGAC & CTTTATGGAAACACAGGCAACA & 103 \\
$\beta$ - actin & TGACGTGGACATCCGCAAAG & CTGGAAGGTGGACAGCGAGG & 205 \\
\hline
\end{tabular}

validation. Proteins with probability based MOWSE scores exceeding their threshold $(\mathrm{P}<0.05)$ were considered to be positively identified.

Western blot analysis. Western blot assay was performed after $10 \%$ SDS-PAGE separation, transfer to a PVDF membrane (Millipore) and saturation with 5\% non-fat dry milk for $2 \mathrm{~h}$ at room temperature. Followed by incubation with primary rabbit polyclonal antibodies including anti-P-gp (1:500 dilution; GeneTex), anti-MRP1 (1:600 dilution; GeneTex), anti-RFC (1:1,500 dilution; Abcam), anti-hnRNP C1/C2 (1:600 dilution; GeneTex), rabbit monoclonal antibodies including anti-BCRP (1:800 dilution; Epitomics), anti-DHFR (1:20,000 dilution; Epitomics), anti-NPM (1:1,000 dilution; Epitomics), anti-ENO1 (1:2,500 dilution; Epitomics), anti-VIM (1:600 dilution; Epitomics), anti-PGAM1 (1:1,000 dilution; Epitomics), anti-PSMA2 (1:2,000 dilution; Epitomics), or anti- $\beta$-actin (1:800 dilution; Beijing Biosynthesis Biotechnology, China) as a loading control overnight at $4^{\circ} \mathrm{C}$, then the membranes were incubated with horseradish peroxidase-conjugated goat anti-rabbit IgG (1:20,000 dilution; CWbiotech, China) for $2 \mathrm{~h}$ at room temperature. Protein bands were detected by ECL detection system. Images were analyzed using a quantitative analysis system (Image-Pro Plus).

Quantitative PCR analysis. Total RNA was extracted using RNAfast2000 kit (Fastagen) according to the manufacturer's instructions. Quantitative PCR was performed with PrimeScript RT Master Mix Perfect Real Time Kit (Takara DRR036A) and SYBR Premix Ex Taq II (Takara). The primer sequences and product length are listed in Table I. The experiments were run on the Bio-Rad CFX96 ${ }^{\mathrm{TM}}$ Real-time system (Bio-Rad): pre-degeneration for $95^{\circ} \mathrm{C}, 30 \mathrm{sec}, 1 \mathrm{cycle}$, and PCR reaction, $95^{\circ} \mathrm{C} 5 \mathrm{sec}$ followed by $60^{\circ} \mathrm{C}, 30 \mathrm{sec}, 40$ cycles, and $95^{\circ} \mathrm{C}$ for $15 \mathrm{sec}, 60^{\circ} \mathrm{C}$ for $30 \mathrm{sec}, 95^{\circ} \mathrm{C}$ for $15 \mathrm{sec}$ for dissociation. $\beta$-actin was used as an internal control gene to normalize expression levels.

Small interfering RNA (siRNA) transfection. MCF-7/MTX cells were seeded in a 6 -well plate at a density of $5 \times 10^{5}$ cells per well in RPMI-1640 without antibiotics. After $24 \mathrm{~h}$, the NPM or non-specific control siRNA (Shanghai GenePharma, China) was transfected with Lipofectamine 2000 (Invitrogen) according to the manufacturer's instructions. The concentration of NPM siRNA was $50 \mathrm{nM}$. The efficiency of RNA interference was checked by quantitative PCR and western blot analysis, respectively. The transfected cells were used for proliferation assay, other genes and proteins detection.

Statistical analysis. All experiments were carried out independently three times. All data, unless stated otherwise, are shown as means \pm standard deviations. Statistical analyses of differences between the groups were performed by Student's t-test. A P-value of $<0.05$ was considered statistically significant.

\section{Results}

Methotrexate-resistant cell line. After a continuous induction with low concentration of MTX in a gradually increasing manner for 12 months, the MCF-7/MTX-resistant cell line grew steadily in the culture medium with $220 \mathrm{nM}$ MTX. The $\mathrm{IC}_{50}$ values of MTX for MCF-7/S cells and MCF-7/MTX cell lines were $44 \pm 4.25 \mathrm{nM}$ and $2818 \pm 97.9 \mathrm{nM}$, respectively (Fig. 1). The resistance of MCF-7/MTX cells to MTX was 64-fold higher than that of MCF-7/S cells, which implied the MTX-resistant cell line was successfully established.

MDR phenotype of MCF-7/MTX cells. Both MCF-7/S and MCF-7/MTX cell lines were treated with different concentrations of chemotherapeutic drugs, and the $\mathrm{IC}_{50}$ and $\mathrm{RF}$ values are summarized in Table II. The cross-resistance of MCF-7/ MTX cells to doxorubicin, paclitaxel, 5-fluorouracil, mitoxantrone, vinorelbine, cisplatin, docetaxel, pemetrexed and gemcitabine significantly increased compared with that of MCF-7/S cells, which indicated the typical MDR phenotype of MCF-7/MTX cells.

In addition, in order to detect whether the MDR of MCF-7/ MTX cells was associated with the classic resistance-related proteins and resistance mechanism of MTX, we checked the 


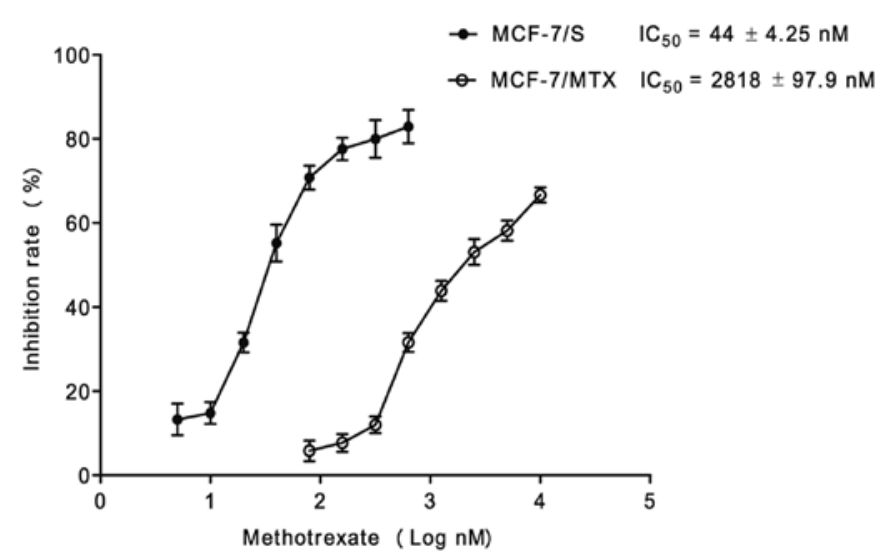

Figure 1. MCF-7/MTX cell line is resistant to methotrexate. MCF-7/S and MCF-7/MTX cells were exposed to methotrexate at the indicated increasing concentrations for $72 \mathrm{~h}$. Dose-inhibition rates were detected by MTT assay. Each value represents the mean \pm SD of eight measurements.

mRNA and protein levels of MDR1, MRP1, BCRP, RFC and DHFR by quantitative PCR and western blot analyses. The mRNA levels of MDR1, MRP1, BCRP and DHFR in MCF-7/ MTX cells were higher than those in MCF-7/S cells, while the mRNA expression of RFC was significant decreased in MCF-7/MTX cell line (Fig. 2A). The protein levels of these molecules were consistent with gene expressions in both cell lines (Fig. 2B). These results demonstrated that the established MCF-7/MTX cell line is a valuable model to study MDR mechanism.

Cell cycle distribution. In order to further explore the effect of MTX on cells, the cell cycle profiles of MCF-7/S and MCF-7/ MTX was performed using flow cytometry analysis. We found that the proportion of MCF-7/MTX cells in G0/G1 and G2/M phases showed a gradual increasing trend, from 63.37 to $65.69 \%$ and from 11.78 to $19.23 \%$, respectively, while the proportion of $\mathrm{S}$ phase dropped from 24.85 to $15.07 \%$ (Fig. 3).
These results suggested that MTX could block the cell cycle at the $\mathrm{G} 0 / \mathrm{G} 1$ phase of progression.

The proteome comparison of MCF-7/MTX and MCF-7/S cells. To compare the proteome between MCF-7/MTX and MCF-7/S cells, we performed 2-DE to separate the global protein lysates of these two cell lines. The analysis was carried out in six gels made from three independent protein samples of each cell line. The images of protein spots were assessed using PDQuest6.0 analysis software. We selected 25 differentially expressed protein spots ( $\geq 1.5$-fold) between these two cell lines for further identification using MALDI-TOF-MS, and 17 proteins that were successively identified were marked with arrows in Fig. 4A, among which 7 proteins were upregulated and 10 proteins were downregulated in the MCF-7/MTX cell line (Table III).

Validation of protein expression. To further confirm the expression of identified proteins, western blotting was performed to validate six differentially expressed proteins (Fig. 4B). Consistent with the results of 2-DE analysis, three proteins nucleophosmin (NPM), $\alpha$-enolase (ENO1), and vimentin (VIM) were significantly upregulated, while other three proteins including heterogeneous nuclear ribonucleoprotein (hnRNP C1/C2), phosphoglycerate mutase 1 (PGAM1), and proteasome subunit $\alpha$ type-2 (PSMA2) were obviously downregulated in MCF-7/MTX cells (Fig. 5A).

Validation of $m R N A$ expression. To further validate whether the mRNA levels of these six differentially expressed proteins had variations, quantitative PCR assay was applied to detect the mRNA expression of NPM, ENO1, VIM, hnRNP Cl/C2, $P G A M 1$ and PSMA2 (Fig. 5B). Compared with MCF-7/S cells, the mRNA levels of $N P M$ and $V I M$ were increased while hnRNP C1/C2, PGAM1 and PSMA2 genes were decreased in $\mathrm{MCF}-7 / \mathrm{MTX}$ cell line, which was consistent with the alteration of the protein levels. Unexpectedly, the mRNA expression of

Table II. $\mathrm{IC}_{50}$ values $(\mathrm{nM})$ of different cells against selected anticancer drugs (mean $\left.\pm \mathrm{SD}\right)$.

\begin{tabular}{|c|c|c|c|c|c|}
\hline \multirow[b]{2}{*}{ Drugs } & \multirow[b]{2}{*}{$\mathrm{MCF}-7 / \mathrm{S}$} & \multirow[b]{2}{*}{ MCF-7/MTX } & \multirow[b]{2}{*}{ MCF-7/MTX RNAi } & \multirow{2}{*}{$\frac{\mathrm{IC}_{50 \mathrm{MCF}-7 / \mathrm{MTX}} / \mathrm{IC}_{50 \mathrm{MCF}-7 / \mathrm{S}}}{\mathrm{RF}}$} & \multirow{2}{*}{$\frac{\mathrm{IC}_{50 \text { MCF-7/MTX RNAi }} / \mathrm{IC}_{50 \text { MCF-7/ }}}{\mathrm{RF}}$} \\
\hline & & & & & \\
\hline Methotrexate & $44 \pm 4.25$ & $2,818 \pm 97.9^{\mathrm{a}}$ & $1,288 \pm 29.0^{\mathrm{b}}$ & 64.0 & 29.3 \\
\hline Doxorubicin & $71 \pm 2.57$ & $245 \pm 7.39^{a}$ & $174 \pm 5.42^{\mathrm{b}}$ & 3.45 & 2.45 \\
\hline Paclitaxel & $20 \pm 0.45$ & $48 \pm 1.62^{\mathrm{a}}$ & $33 \pm 1.05^{\mathrm{b}}$ & 2.40 & 1.65 \\
\hline 5-fluorouracil & $3,890 \pm 178$ & $7,943 \pm 55.3^{\mathrm{a}}$ & $6,892 \pm 57.9^{\mathrm{b}}$ & 2.04 & 1.77 \\
\hline Mitoxantrone & $186 \pm 16.0$ & $295 \pm 24.8^{\mathrm{a}}$ & $285 \pm 21.0$ & 1.58 & 1.53 \\
\hline Vinorelbine & $51 \pm 7.18$ & $1,023 \pm 91.6^{\mathrm{a}}$ & $256 \pm 10.4^{b}$ & 20.1 & 5.02 \\
\hline Cisplatin & $2,042 \pm 74.2$ & $5,495 \pm 66.2^{\mathrm{a}}$ & $3,987 \pm 187^{\mathrm{b}}$ & 2.69 & 1.95 \\
\hline Docetaxel & $4.27 \pm 0.19$ & $22 \pm 5.42^{\mathrm{a}}$ & $14 \pm 1.87^{\mathrm{b}}$ & 5.15 & 3.28 \\
\hline Pemetrexed & $3,631 \pm 216$ & $9,550 \pm 111^{\mathrm{a}}$ & $6,424 \pm 245^{\mathrm{b}}$ & 2.63 & 1.77 \\
\hline Gemcitabine & $955 \pm 47.0$ & $6,026 \pm 568^{a}$ & $2,698 \pm 109^{b}$ & 6.31 & 2.83 \\
\hline
\end{tabular}

MCF-7/MTX versus MCF-7/S; ${ }^{a} \mathrm{P}<0.01 . \mathrm{MCF}-7 / \mathrm{MTX}$ RNAi versus MCF-7/MTX; ${ }^{\mathrm{b}} \mathrm{P}<0.01$. 
A

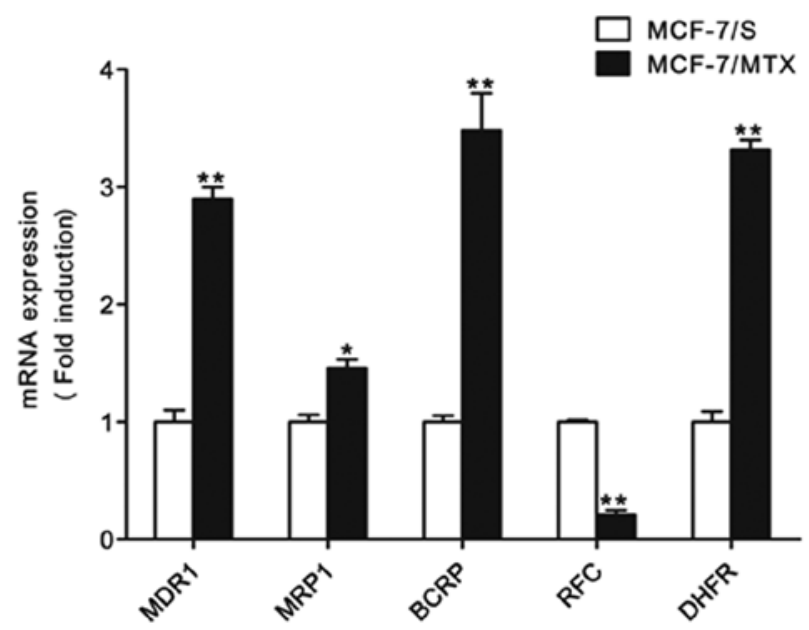

B
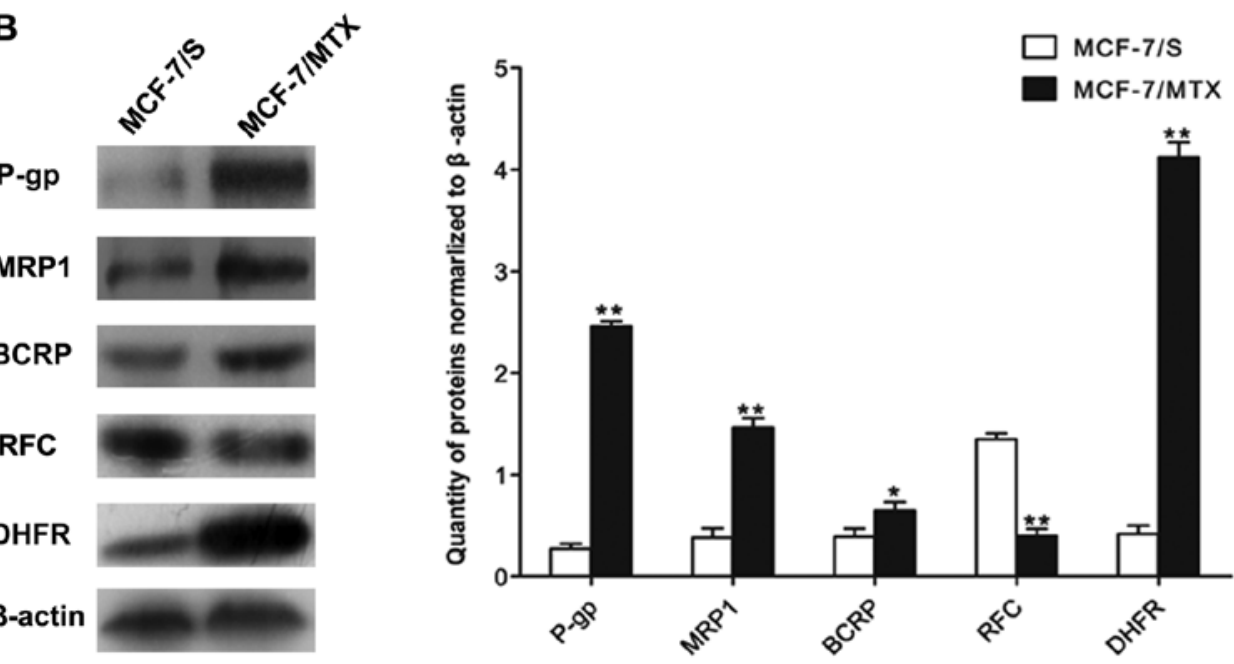

Figure 2. MCF-7/MTX cells have classic MDR and MTX resistance. (A) The mRNA expression of MDR1, MRP1, BCRP, RFC and DHFR in MCF-7/S and MCF-7/MTX cells was compared by quantitative PCR. The results are shown by fold change ( \pm SEM) relative to the control. (B) The protein levels of P-gp, MRP1, BCRP, RFC and DHFR in MCF-7/S and MCF-7/MTX cells were detected by western blot analyses (left panel). Relative expression is shown as mean $\pm \mathrm{SD}$ (right panel). All experiments were performed three times $\left({ }^{*} \mathrm{P}<0.05 ;{ }^{* *} \mathrm{P}<0.01\right)$.

MCF-7/S

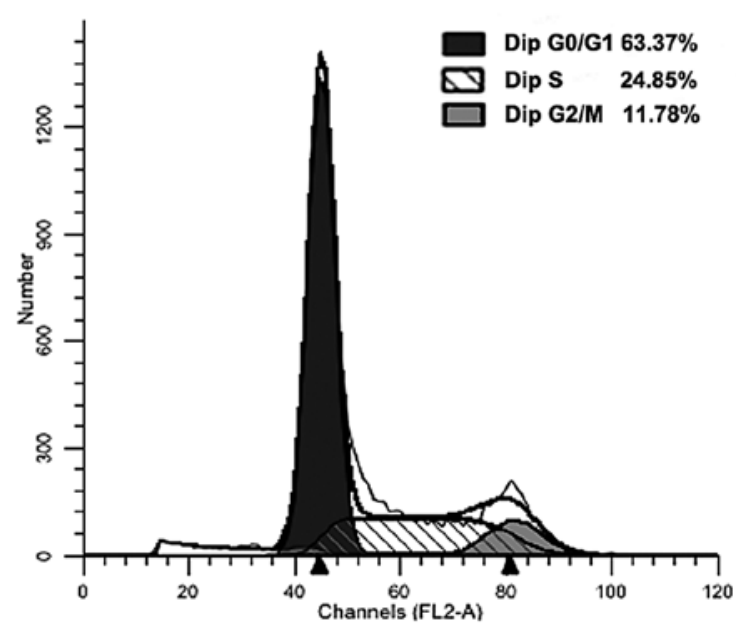

MCF-7/MTX

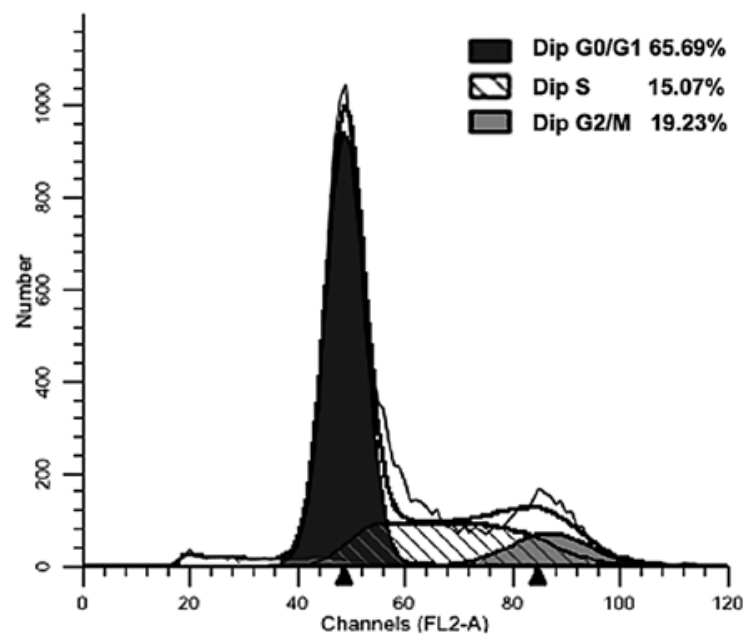

Figure 3. Cell cycle distribution of MCF-7/S and MCF-7/MTX cells. The DNA content was evaluated by flow cytometry after ethanol fixation and PI staining. Data are represented as proportion of cells in $\mathrm{G} 0 / \mathrm{G} 1, \mathrm{~S}$ and $\mathrm{G} 2 / \mathrm{M}$ phases of the cell cycle from three independent experiments. 
A

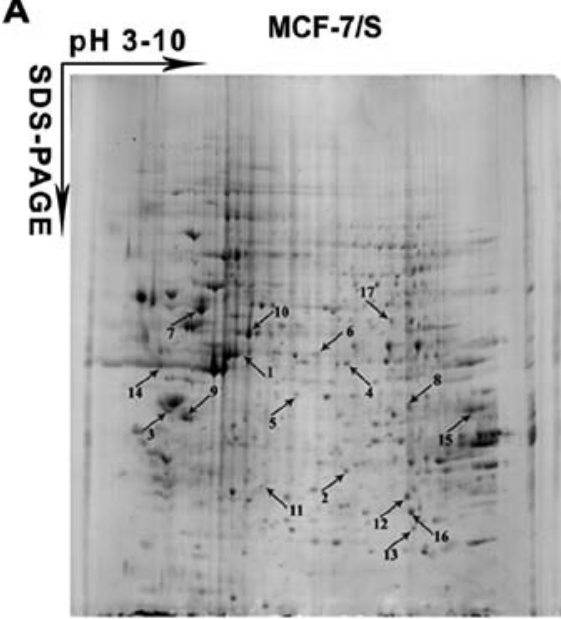

B
MCF-7/MTX

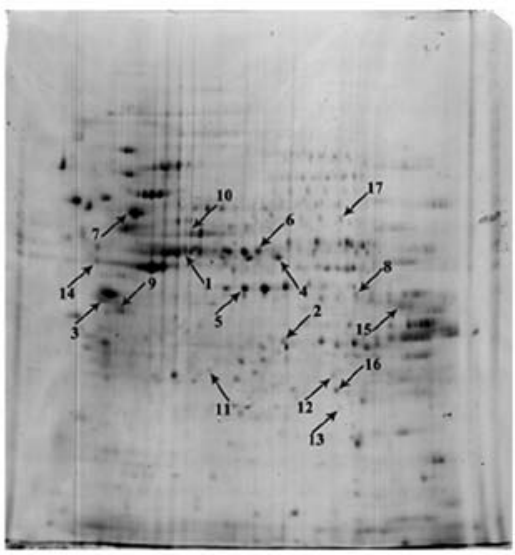

MCF-7/MTX
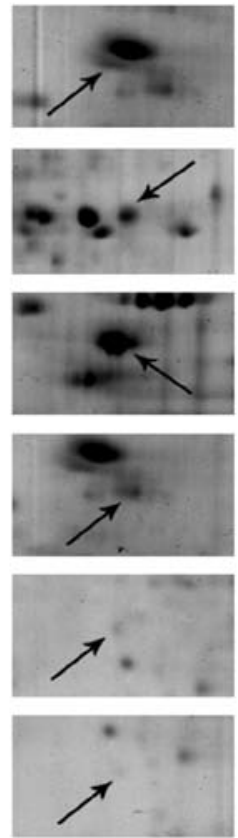

Figure 4. Proteomic mapping of differentially expressed proteins from MCF-7/S and MCF-7/MTX cells. (A) Comparison of the protein gels between MCF-7/ MTX and MCF-7/S cells. The 17 differentially expressed protein spots were labeled with arrows and numbered for MS analysis. (B) Close-up view of the six differentially expressed protein spots.

ENO1 was slightly downregulated in the MCF-7 MTX cells, which was different from the protein level.

Reversal of MDR in MCF-7/MTX cells by knockdown of NPM. We found the protein and mRNA expressions of NPM were both significantly upregulated in the MCF-7/MTX cells. To study the functions of the increased expression of NPM in the MDR of MCF-7/MTX cells, MTT assay was performed to determine the sensitivity of MCF-7/MTX cells to MTX and other antitumor drugs following knockdown of NPM by siRNA. Our results showed that siRNA significantly downregulated the gene and protein of NPM in the MCF-7/MTX cells (Fig. 6A). Knockdown of NPM by siRNA in MCF-7/ MTX cells obviously enhanced the inhibitory effect of MTX on proliferation. In addition, the $\mathrm{IC}_{50}$ value and $\mathrm{RF}$ of MCF7/ MTX cells exposed to doxorubicin, paclitaxel, 5-fluorouracil, vinorelbine, cisplatin, docetaxel, pemetrexed and gemcitabine were significantly decreased after downregulating the expression of NPM by siRNA (Table II). Surprisingly, the protein and mRNA levels of drug resistance-related molecules, MDR1, MRP1, BCRP and DHFR were also significantly decreased while the protein and gene expressions of RFC were increased after knockdown of NPM in MCF-7/MTX cells (Fig. 6B). These observations demonstrate that NPM has a prominent role in the multidrug resistance of MCF-7/MTX cells.

\section{Discussion}

The antifolate MTX is applied to the treatment of a wide variety of cancers, it is usually given in combination with cyclophosphamide and 5-flurouracil to cure breast cancer. However, drug resistance is frequently observed upon treat- 
A
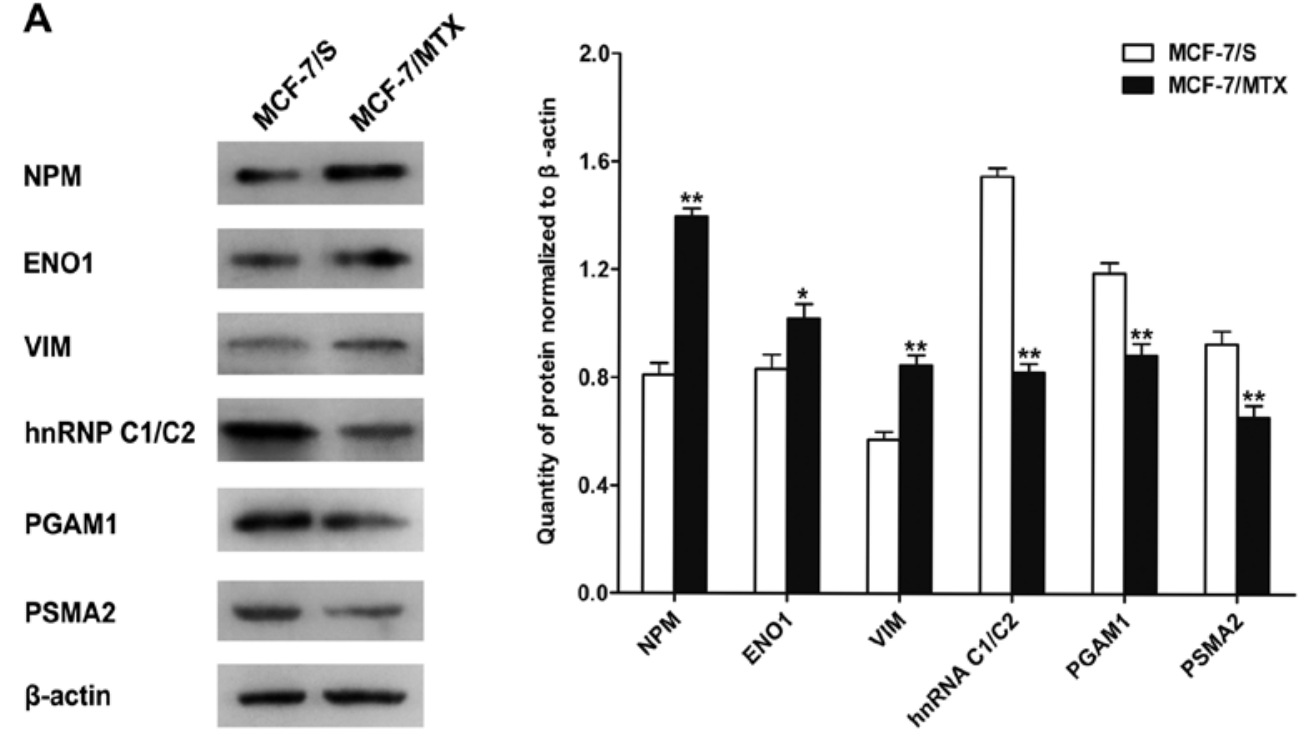

B

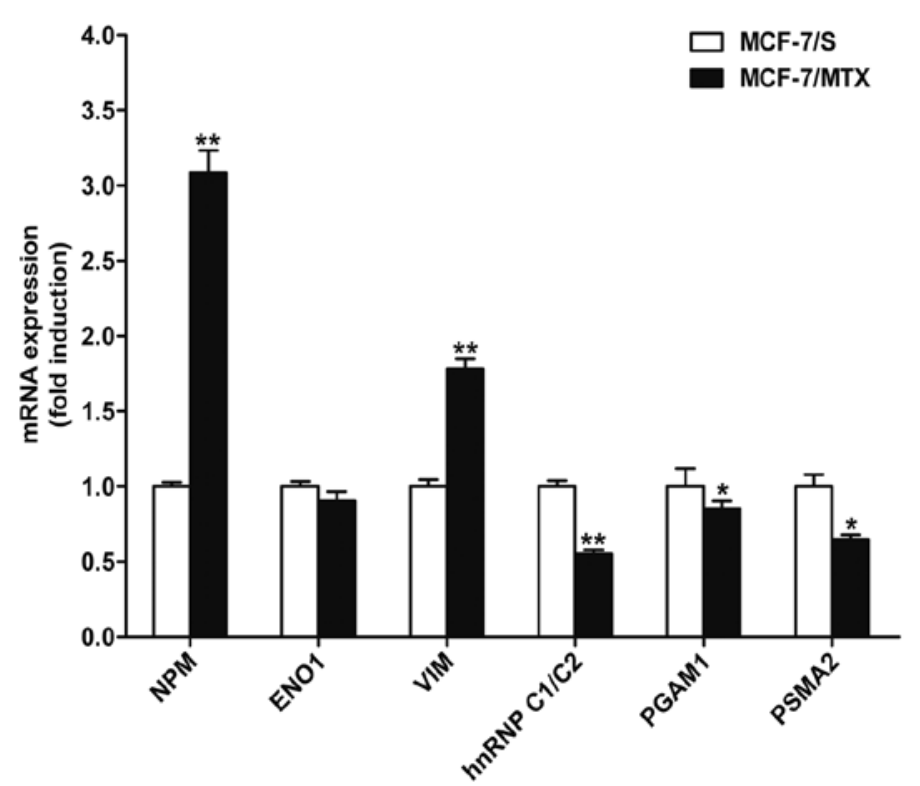

Figure 5. Validation of protein and mRNA levels of NPM, ENO1, VIM, hnRNPC1/C2, PGAM1 and PSMA2 between MCF-7/MTX and MCF-7/S cells. (A) Representative proteins from MCF-7/S and MCF-7/MTX cells were analyzed by western blotting (left panel). The histograms represent the relative density of protein expression by normalizing against $\beta$-actin (right panel). (B) The mRNAs were compared between the two cell lines by quantitative PCR analysis. The results are shown by fold change $( \pm$ SEM) relative to the control. MCF-7/S cells served as control. All experiments were repeated three times $\left({ }^{*} \mathrm{P}<0.05 ;{ }^{* *} \mathrm{P}<0.01\right)$.

ment with MTX for cancer patients, thus compromising the drug effectiveness. Although the alteration of gene expressions also contribute to MTX resistance, such as increase in S100A4, UGT1A6, caveolin-1, enolase-2, PRKCA and the decrease of miR-224 or E-cadherin (7-10), the resistance mechanism of MTX, especially the MDR caused by MTX is not fully understood. In the present study, we have successfully established the MTX-resistant human breast cancer cell line MCF-7/MTX, which displays cross-resistance to a variety of commonly used chemotherapy drugs and multidrug resistance phenotype. Using the proteomic assay, we integrally analyzed the protein expression differences between resistant cells and parental cells to identify potential molecular targets for MDR of breast cancer.
In this study, 17 differentially expressed proteins were identified, seven proteins were upregulated and 10 proteins were downregulated in MCF-7/MTX cells. Moreover, six significantly expressed proteins with distinct functions were validated by western blot and quantitative PCR assays at protein and mRNA levels, respectively. These proteins are involved in tumorigenesis, metabolism, glycolysis, cell proliferation, apoptosis and the invasion process. Their roles in the formation of drug resistance and molecular mechanism are discussed below.

$\alpha$-enolase (ENO1), known as 2-phosphate-D-glycerate hydrolase, catalyzes the formation of phosphoenolpyruvate from 2-phosphoglycerate in the process of glycolysis, which was a key role in cellular energy metabolism (11). 
Table III. MS identification of differentially expressed proteins between MCF-7/MTX and MCF-7/S cells.

\begin{tabular}{|c|c|c|c|c|c|c|c|}
\hline $\begin{array}{l}\text { Spot } \\
\text { no. }\end{array}$ & $\begin{array}{l}\text { Accession } \\
\text { no. }\end{array}$ & Protein name & $\begin{array}{l}\mathrm{MW}(\mathrm{kDa}) / \\
\text { PI }\end{array}$ & $\begin{array}{l}\text { Peptide } \\
\text { matched }\end{array}$ & Score & $\begin{array}{c}\text { Sequence } \\
\text { coverage } \\
(\%)\end{array}$ & $\begin{array}{c}\text { Expression in } \\
\text { MCF-7/MTX/ } \\
\text { MCF-7/S }\end{array}$ \\
\hline 1 & IPI00554788 & Keratin, type I cytoskeletal 18 & $48.03 / 5.34$ & 26 & 661 & 49 & $4.43 \uparrow$ \\
\hline 2 & IPI00645031 & Isoform 2 of $\lambda$-crystallin homolog & $33.79 / 5.68$ & 10 & 66 & 24 & $4.15 \uparrow$ \\
\hline 3 & IPI00658013 & Nucleophosmin isoform 3 & $28.50 / 4.56$ & 8 & 168 & 33 & $4.05 \uparrow$ \\
\hline 4 & IPI00893990 & NDUFA10 protein & $15.90 / 5.14$ & 9 & 73 & 46 & $3.58 \uparrow$ \\
\hline 5 & IPI00022465 & $\begin{array}{l}\text { Isoform } 1 \text { of citron } \\
\text { Rho-interacting kinase }\end{array}$ & $233.34 / 6.16$ & 22 & 65 & 11 & $2.61 \uparrow$ \\
\hline 6 & IPI00465248 & Isoform $\alpha$-enolase of $\alpha$-enolase & $47.48 / 7.01$ & 16 & 277 & 39 & $2.43 \uparrow$ \\
\hline 7 & IPI00418471 & Vimentin & $53.68 / 5.06$ & 23 & 663 & 53 & $2.08 \uparrow$ \\
\hline 8 & IPI00016610 & Poly(rC)-binding protein 1 & $37.99 / 6.66$ & 13 & 450 & 50 & $3.48 \downarrow$ \\
\hline 9 & IPI00216592 & $\begin{array}{l}\text { Isoform } \mathrm{C} 1 \text { of heterogeneous nuclear } \\
\text { ribonucleoproteins } \mathrm{C} 1 / \mathrm{C} 2\end{array}$ & $32.37 / 4.94$ & 14 & 624 & 43 & $3.34 \downarrow$ \\
\hline 10 & IPI00554648 & Keratin, type II cytoskeletal 8 & $53.67 / 5.52$ & 24 & 665 & 49 & $3.10 \downarrow$ \\
\hline 11 & IPI00024911 & $\begin{array}{l}\text { Endoplasmic reticulum resident } \\
\text { protein } 29\end{array}$ & $29.03 / 6.77$ & 7 & 173 & 33 & $2.92 \downarrow$ \\
\hline 12 & IPI00549725 & Phosphoglycerate mutase 1 & $28.90 / 6.67$ & 15 & 895 & 62 & $2.79 \downarrow$ \\
\hline 13 & IPI00219622 & Proteasome subunit $\alpha$ type- 2 & $26.00 / 6.92$ & 10 & 222 & 41 & $2.69 \downarrow$ \\
\hline 14 & IPI00739539 & $\begin{array}{l}\text { POTE ankyrin domain family } \\
\text { member F }\end{array}$ & $123.02 / 5.83$ & 13 & 386 & 47 & $2.37 \downarrow$ \\
\hline 15 & IPI00465439 & Fructose-bisphosphatealdolase A & $39.85 / 8.30$ & 14 & 386 & 44 & $2.12 \downarrow$ \\
\hline 16 & IPI00797270 & Isoform 1 of triosephosphateisomerase & $26.94 / 6.45$ & 19 & 682 & 90 & $1.83 \downarrow$ \\
\hline 17 & IPI00163187 & Fascin & $55.12 / 6.84$ & 13 & 361 & 34 & $1.81 \downarrow$ \\
\hline
\end{tabular}

ENO1 has been recognized as a conserved and single function protein, but recent studies found that the effect of this enzyme is involved in transcription, regulation of apoptosis and cell differentiation processes. Research has shown that the expression of ENO1 significantly increased in certain tumor cells, including liver cancer, lung cancer, head and neck cancer, which could be used as prognostic indicators for clinical treatment (12-14). It was reported that the elevated expression of ENO1 was closely related to tamoxifen and adriamycin resistance in breast cancer $(15,16)$. The expression of ENO1 was regulated by ERK1/2 appearing to be mediated by c-Myc, which changed the level of extracellular ATP, thus affecting cell survival (17). In our study, the protein level of ENO1 in MCF-7/MTX cells are significantly increased, but its gene level was slightly lower in the resistant cells, which indicates the mRNA level is not fully present in the protein level, due to the mRNA possessed storage, transport, degradation, translational regulation and post-translational processing of the product, which affects the quality and quantity of the protein (18). We have found the correlation between ENO1 and MDR in breast cancer, which may be regulated by activating ERK1/2 pathway, further affecting tumor cell proliferation.
Vimentin (VIM) is a member of intermediate filament protein family, which constitutes the cytoskeleton with microtubules and actin filaments, it is involved in cell adhesion, migration, apoptosis and cell signal transduction (19). In prostate cancer and triple-negative breast cancer cells, the high expression of VIM was significantly associated with cell invasiveness, moreover, the activation of ERK signaling pathway promotes the overexpression of VIM and increased cell migration and invasion in head and neck cancer (20-22). In recent research, VIM was intimately associated with the resistance to chemotherapeutic drugs, it was significantly higher expression in resistant cancer cells including paclitaxel-resistant prostate cancer, temozolomide-resistant malignant glioma and tamoxifen-resistant breast cancer (23-25). Our results show that the protein and mRNA levels of VIM are significantly increased in the MTX-resistant cells, which may be related to the variation of cell adhesion, thereby possibly influencing cell metastasis and invasion, and its resistance mechanisms need to be further explored.

Heterogeneous nuclear ribonucleoprotein $\mathrm{C} 1 / \mathrm{C} 2$ (hnRNP $\mathrm{C} 1 / \mathrm{C} 2$ ) is a member of hnRNPs family, as an RNA-binding protein, it participates in the regulation of pre-mRNA splicing and post-translational modification (26). In addition, studies 
A

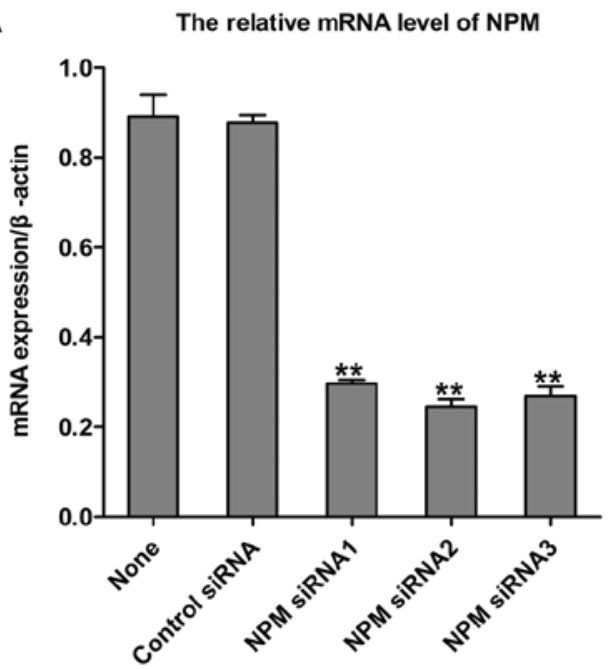

B

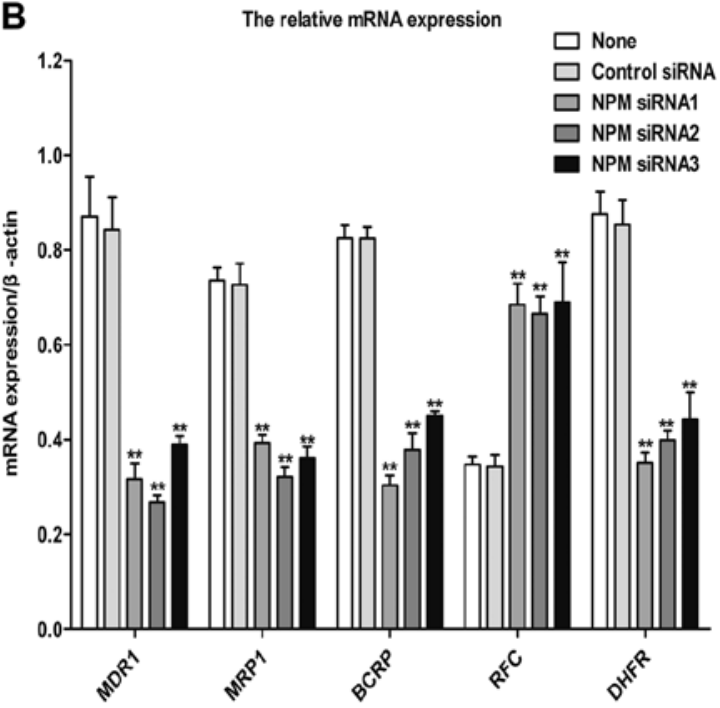

NPM

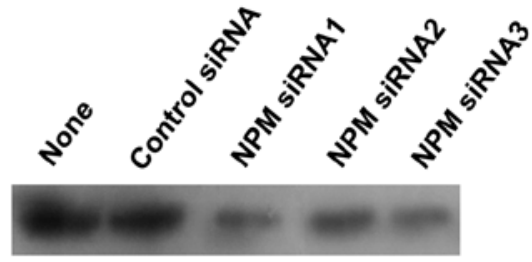

$\beta$-actin
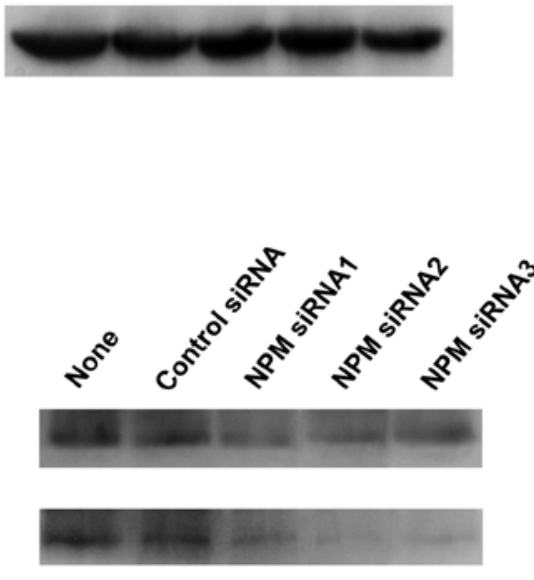

BCRP

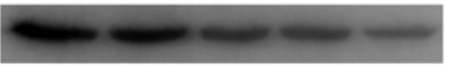

RFC

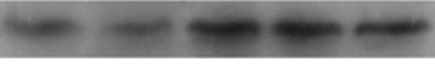

DHFR

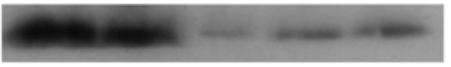

$\beta$-actin

Figure 6. Knockdown of NPM attenuates MDR in the MCF-7/MTX cell line. (A) Quantitative PCR and western blot assay mRNA (left panel) and protein (right panel) levels of NPM in MCF-7/MTX cell line following three siRNA transfections. (B) The mRNA (left panel) and protein (right panel) levels of MDR1, MRP1, BCRP, RFC and DHFR were examined in MCF-7/MTX cells of NPM knockdown by quantitative PCR and western blot analysis. A negative control was used as a control siRNA. All experiments were repeated in triplicate $\left({ }^{*} \mathrm{P}<0.05 ;{ }^{* *} \mathrm{P}<0.01\right)$.

have shown that hnRNPs were associated with tumor development and prognosis (27). Previously, hnRNP C1/C2 has been considered as an apoptosis-related protein, and the protein level of hnRNP C1/C2 was p53-dependently upregulated, but its gene transcription did not depend on the p53 role in colon cancer cells after mitomycin C treatment $(28,29)$. Furthermore, in A549 lung cancer cells of wild-type p53, the expression of $\mathrm{hnRNP} \mathrm{C1/C2}$ was significantly reduced in the process of apoptosis, it regulated the synthesis of p53 isoforms with p53 IRES interaction and affected tumor cell death $(30,31)$. On the contrary, hnRNP C1/C2 was associated with DNA repair function, knockdown of hnRNP $\mathrm{Cl} / \mathrm{C} 2$ by RNA interference could increase the sensitivity of HeLa cells to chemotherapeutic drugs (32). In the present study, both protein and mRNA expression of hnRNP C1/C2 are downregulated in MCF-7/MTX cells, which may be connected with mutations of apoptosis regulatory factors and affects the normal process of cell apoptosis, while the exact mechanism needs further investigation.
Phosphoglycerate mutase 1 (PGAM1), as a key enzyme of glycolysis and gluconeogenesis process, catalyzes the conversion of 3-phosphoglycerate (3-PG) to 2-phosphoglycerate (2-PG). It was found that PGAM1 was overexpressed in certain tumor cells including liver cancer, breast cancer, and glioma, its upregulation may facilitate the proliferation and metabolism of cancer cells, which could be used as a potential therapeutic target for tumors (33-36). Moreover, PGAM1 was also negatively correlated with the tumor suppressor p53, which further illustrates the interaction between PGAM1 and cell apoptosis (37). However, we discovered that the protein and mRNA levels of PGAM1 are significantly reduced in MTX-resistant cells, abnormal glucose metabolism may be involved in mediating the role of MDR in breast cancer, and the resistance mechanism is still unclear and requires further study.

Proteasomes are macromolecular complexes with a variety of proteolytic functions, acting as catalysts in the ubiquitin-proteasome pathway of intracellular protein degradation. However, the ubiquitin-proteasome pathway may control numerous important 
physiological functions, such as cell cycle, signal transduction, DNA damage repair and cell apoptosis, additionally, abnormal alteration of this pathway is closely bound with prognosis and development of malignant tumor. Increased expression of proteasome subunit protein can lead to formation of tumors, especially to protect anti-apoptotic effects of cancer cells, therefore, the proteasome subunit proteins could be recognized as potential antitumor targets. Some studies demonstrated that PSMA7 could inhibit proliferation and invasion in A549 cells (38), it regulated cell transcription, cell cycle and tumor development with other important proteins interaction (39). It was found that PSMA5 was highly expressed in 5-fluorouracil-resistant colorectal cancer cells, which showed certain anti-apoptotic activity (40). Whereas, in our established MTX-resistant cell line, we first discover that the protein and gene levels of PSMA2 are significantly downregulated, which reflects a correlation between PSMA2 and MDR in breast cancer, this provides the basis for future investigation of resistance mechanisms.

Nucleolar phosphoprotein (NPM) is abundant and highly conserved phosphoprotein which shuttles rapidly between nucleus and cytoplasm, it participates in the assembly and synthesis of ribosome, replication of chromosomes and centrosomes and intracellular signal transduction, which plays an important role in cell growth, proliferation and transformation. An increasing number of studies manifested that there may exist a connection between NPM and the occurence of tumors, high expression of the proto-oncogene c-Myc could lead to upregulation of NPM transcriptional level; its overexpression could inactivate the function of p53 and enhance the anti-apoptosis effect in cancer cells; NPM was able to regulate tumor progression by activating the phosphorylation of MAPK/ ERK and c-Myc signaling pathway (41). The overexpression of NPM defended the p53-mediated cellular senescence and growth arrest in colorectal cancer cells, which showed its role in promoting tumor growth (42), conversely, reducing the expression of NPM by shRNA sensitized the resistant leukemia cells to chemotherapy (43). There is also evidence indicating that NPM has a tumor suppressor property maintaining the gene stability and regulating the ARF tumor suppressor (44). Similarly, in invasive breast cancer MDA-MB-231 cells, the overexpression of NPM inhibited cell growth, which could be used as a tumor suppressor factor and predictor of poor prognosis (45). Although the above demonstrated functions of NPM are controversial, in our study, the protein and gene levels of NPM are both significantly increased in MCF-7/MTX cells. We discovered the connection between NPM and MTX resistance mechanism, which may induce MDR through acting on MAPK/ERK signaling pathway in breast cancer. Subsequently, we reduced the expression of NPM by siRNA interference, which attenuates the resistance of MCF-7/MTX cells to various drugs and the expressions of cell factors related to MDR and MTX resistance mechanism. These results indicate that NPM contributes to MTX resistance in breast cancer cells and suggest that NPM may play a significant role in the occurrence of MDR of cancer cells.

In conclusion, we have successfully established the MTX-resistant human breast cancer cell line and identified 17 differentially expressed proteins. NPM, involving in tumorigenesis and signal transduction, plays a prominent role in the MDR of MCF-7/MTX cells. This study investigated the resistance mechanisms of breast cancer and provided a theoretical basis for the clinical diagnosis of MDR.

\section{Acknowledgements}

This study was supported by National Natural Science Foundation of China (nos. 30973673 and 30973578).

\section{References}

1. Jemal A, Bray F, Center MM, Ferlay J, Ward E and Forman D: Global cancer statistics. CA Cancer J Clin 61: 69-90, 2011.

2. Bhoo-Pathy N, Yip CH, Hartman M, et al: Breast cancer research in Asia: adopt or adapt Western knowledge? Eur J Cancer 49: 703-709, 2013.

3. Longley DB and Johnston PG: Molecular mechanisms of drug resistance. J Pathol 205: 275-292, 2005.

4. Baguley BC: Multiple drug resistance mechanisms in cancer. Mol Biotechnol 46: 308-316, 2010.

5. Olsen EA: The pharmacology of methotrexate. J Am Acad Dermatol 25: 306-318, 1991.

6. Assaraf YG: Molecular basis of antifolate resistance. Cancer Metastasis Rev 26: 153-181, 2007.

7. de Almagro MC, Selga E, Thibaut R, Porte C, Noe V and Ciudad CJ: UDP-glucuronosyltransferase 1A6 overexpression in breast cancer cells resistant to methotrexate. Biochem Pharmacol 81: 60-70, 2011.

8. Selga E, Morales C, Noe V, Peinado MA and Ciudad CJ: Role of caveolin 1, E-cadherin, Enolase 2 and PKCalpha on resistance to methotrexate in human HT29 colon cancer cells. BMC Med Genomics 1: 35, 2008.

9. Mencia N, Selga E, Noé V and Ciudad CJ: Underexpression of miR-224 in methotrexate resistant human colon cancer cells. Biochem Pharmacol 82: 1572-1582, 2011.

10. Mencia N, Selga E, Rico I, et al: Overexpression of S100A4 in human cancer cell lines resistant to methotrexate. BMC Cancer 10: $250,2010$.

11. Subramanian A and Miller DM: Structural analysis of alpha-enolase. Mapping the functional domains involved in down-regulation of the c-myc protooncogene. J Biol Chem 275: 5958-5965, 2000.

12. Takashima M, Kuramitsu Y, Yokoyama Y, et al: Overexpression of alpha enolase in hepatitis $\mathrm{C}$ virus-related hepatocellular carcinoma: association with tumor progression as determined by proteomic analysis. Proteomics 5: 1686-1692, 2005.

13. Chang GC, Liu KJ, Hsieh CL, et al: Identification of alpha-enolase as an autoantigen in lung cancer: its overexpression is associated with clinical outcomes. Clin Cancer Res 12: 5746-5754, 2006.

14. Tsai ST, Chien IH, Shen WH, et al: ENO1, a potential prognostic head and neck cancer marker, promotes transformation partly via chemokine CCL20 induction. Eur J Cancer 46: 1712-1723, 2010.

15. Tu SH, Chang CC, Chen CS, et al: Increased expression of enolase alpha in human breast cancer confers tamoxifen resistance in human breast cancer cells. Breast Cancer Res Treat 121: 539-553, 2010.

16. Chuthapisith S, Layfield R, Kerr ID, Hughes C and Eremin O: Proteomic profiling of MCF-7 breast cancer cells with chemoresistance to different types of anti-cancer drugs. Int J Oncol 30: 1545-1551, 2007.

17. Mizukami Y, Iwamatsu A, Aki T, et al: ERK1/2 regulates intracellular ATP levels through alpha-enolase expression in cardiomyocytes exposed to ischemic hypoxia and reoxygenation. J Biol Chem 279: 50120-50131, 2004.

18. de Sousa Abreu R, Penalva LO, Marcotte EM and Vogel C: Global signatures of protein and mRNA expression levels. Mol Biosyst 5: 1512-1526, 2009.

19. Ivaska J, Pallari HM, Nevo J and Eriksson JE: Novel functions of vimentin in cell adhesion, migration, and signaling. Exp Cell Res 313: 2050-2062, 2007.

20. Singh S, Sadacharan S, Su S, Belldegrun A, Persad S and Singh G: Overexpression of vimentin: role in the invasive phenotype in an androgen-independent model of prostate cancer. Cancer Res 63: 2306-2311, 2003

21. Karihtala P, Auvinen P, Kauppila S, Haapasaari KM, JukkolaVuorinen A and Soini Y: Vimentin, zeb1 and Sipl are up-regulated in triple-negative and basal-like breast cancers: association with an aggressive tumour phenotype. Breast Cancer Res Treat 138: 81-90, 2013. 
22. Tseng YH, Chang KW, Yang CC, et al: Association between areca-stimulated vimentin expression and the progression of head and neck cancers. Head Neck 34: 245-253, 2012.

23. Kim JJ, Yin B, Christudass CS, et al: Acquisition of paclitaxel resistance is associated with a more aggressive and invasive phenotype in prostate cancer. J Cell Biochem 114: 1286-1293, 2013.

24. Sun S, Wong TS, Zhang XQ, et al: Protein alterations associated with temozolomide resistance in subclones of human glioblastoma cell lines. J Neurooncol 107: 89-100, 2012.

25. Liu H, Zhang HW, Sun XF, et al: Tamoxifen-resistant breast cancer cells possess cancer stem-like cell properties. Chin Med J (Engl) 126: 3030-3034, 2013.

26. Krecic AM and Swanson MS: hnRNP complexes: composition, structure, and function. Curr Opin Cell Biol 11: 363-371, 1999.

27. Carpenter B, MacKay C, Alnabulsi A, et al: The roles of heterogeneous nuclear ribonucleoproteins in tumour development and progression. Biochim Biophys Acta 1765: 85-100, 2006.

28. Brockstedt E, Rickers A, Kostka S, et al: Identification of apoptosis-associated proteins in a human Burkitt lymphoma cel line. Cleavage of heterogeneous nuclear ribonucleoprotein A1 by caspase 3. J Biol Chem 273: 28057-28064, 1998.

29. Rahman-Roblick R, Johannes Roblick U, Hellman U, et al: p53 targets identified by protein expression profiling. Proc Natl Acad Sci USA 104: 5401-5406, 2007.

30. Koryllou A, Patrinou-Georgoula M, Troungos C and Pletsa V: Cell death induced by N-methyl-N-nitrosourea, a model SN1 methylating agent, in two lung cancer cell lines of human origin. Apoptosis 14: 1121-1133, 2009.

31. Grover R, Sharathchandra A, Ponnuswamy A, Khan D and Das S: Effect of mutations on the p53 IRES RNA structure: Implications for de-regulation of the synthesis of p53 isoforms RNA Biol 8: 132-142, 2011.

32. Hossain MN, Fuji M, Miki K, Endoh M and Ayusawa D: Downregulation of hnRNP C1/C2 by siRNA sensitizes HeLa cells to various stresses. Mol Cell Biochem 296: 151-157, 2007.

33. Ren F, Wu H, Lei Y, et al: Quantitative proteomics identification of phosphoglycerate mutase 1 as a novel therapeutic target in hepatocellular carcinoma. Mol Cancer 9: 81, 2010.
34. Evans MJ, Saghatelian A, Sorensen EJ and Cravatt BF: Target discovery in small-molecule cell-based screens by in situ proteome reactivity profiling. Nat Biotechnol 23: 1303-1307, 2005.

35. Gao H, Yu B, Yan Y, et al: Correlation of expression levels of ANXA2, PGAM1, and CALR with glioma grade and prognosis. J Neurosurg 118: 846-853, 2013.

36. Jiang X, Sun Q, Li H, Li K and Ren X: The role of phosphoglycerate mutase 1 in tumor aerobic glycolysis and its potential therapeutic implications. Int J Cancer: Nov 28, 2013 (Epub ahead of print). doi: 10.1002/ijc.28637.

37. Chaneton B and Gottlieb E: PGAMgnam style: a glycolytic switch controls biosynthesis. Cancer Cell 22: 565-566, 2012.

38. Tan JY, Huang X and Luo YL: PSMA7 inhibits the tumorigenicity of A549 human lung adenocarcinoma cells. Mol Cell Biochem 366: 131-137, 2012.

39. Du H, Huang X, Wang S, Wu Y, Xu W and Li M: PSMA7, a potential biomarker of diseases. Protein Pept Lett 16: 486-489, 2009.

40. Sakai A, Otani M, Miyamoto A, Yoshida H, Furuya E and Tanigawa N: Identification of phosphorylated serine -15 and -82 residues of HSPB1 in 5-fluorouracil-resistant colorectal cancer cells by proteomics. J Proteomics 75: 806-818, 2012.

41. Yung BY: Oncogenic role of nucleophosmin/B23. Chang Gung Med J 30: 285-293, 2007.

42. Wong JC, Hasan MR, Rahman M, et al: Nucleophosmin 1, upregulated in adenomas and cancers of the colon, inhibits p53-mediated cellular senescence. Int J Cancer 133: 1567-1577, 2013.

43. Lin M, Hu J, Liu T, Li J, Chen B and Chen X: Knockdown of nucleophosmin by RNA interference reverses multidrug resistance in resistant leukemic HL-60 cells. Immunobiology 218: $1147-1154,2013$.

44. Grisendi S, Mecucci C, Falini B and Pandolfi PP: Nucleophosmin and cancer. Nat Rev Cancer 6: 493-505, 2006.

45. Karhemo PR, Rivinoja A, Lundin J, et al: An extensive tumor array analysis supports tumor suppressive role for nucleophosmin in breast cancer. Am J Pathol 179: 1004-1014, 2011. 